\title{
Nonlinear Theory of Collisionless Trapped Ion Modes
}

\author{
T.S. Hahm and W.M. Tang \\ Princeton University, Princeton Plasma Physics Laboratory \\ P.O. Box 451, Princeton, NJ 08543
}

\begin{abstract}
A simplified two field nonlinear model for collisionless trapped-ion-mode turbulence has been derived from nonlinear bounce-averaged drift kinetic equations. The renormalized thermal diffusivity obtained from this analysis exhibits a Bohm-like scaling. A new nonlinearity associated with the neoclassical polarization density is found to introduce an isotopedependent modification to this Bohm-like diffusivity. The asymptotic balance between the equilibrium variation and the finite banana width induced reduction of the fluctuation potential leads to the result that the radial correlation length decreases with increasing plasma current. Other important conclusions from the present analysis include the predictions that (i) the relative density fluctuation level $\delta n / n_{0}$ is lower than the conventional mixing length estimate, $\Delta r / L_{n}$; (ii) the ion temperature fluctuation level $\delta T_{i} / T_{i}$ significantly exceeds the density fluctuation level $\delta n / n_{0}$; and (iii) the parallel ion velocity fluctuation level $\delta v_{i \|} / v_{T i}$ is expected to be negligible.
\end{abstract}

\footnotetext{
${ }^{1}$ PACS numbers:52.35.Ra, 52.25.Gj, 52.25.Fi, 52.55.Fa
} 


\section{Introduction}

Transport scaling trends closer to Bohm $(\chi \propto c T / e B)$ rather than gyro-Bohm $(\chi \propto$ $c T \rho / e B a)$ have been prominently observed in major tokamak experiments such as Tokamak Fusion Test Reactor (TFTR). ${ }^{1}$ Important investigations of this key issue have also been carried out in the DIII-D device. ${ }^{2}$ This is very suggestive of the importance of long wavelength, low frequency fluctuations. Indeed, Beam Emission Spectroscopy (BES) measurements on $\mathrm{TFTR}^{3}$ and the results from the studies using a comprehensive two dimensional (2-D) kinetic code ${ }^{4}$ have shown the existence of significant density fluctuations in the trapped ion mode (TIM) regime corresponding to $\omega$ (mode frequency) $<\omega_{b i}$ (trapped ion bounce frequency).

Motivated by the preceding considerations, a nonlinear analysis of collisionless trapped ion modes (CTIM) has been carried out. These instabilities are expected to be relevant in

the core region of present day large tokamak plasmas characterized by very low collisionality with $\omega>\nu_{e, e f f}$ (effective trapped electron collisional frequency). Although the existence of CTIM in low collisionality high temperature plasmas was predicted almost three decades ago,${ }^{5}$ most of the subsequent nonlinear studies on TIM focused on the dissipative trapped ion mode (DTIM) regime with $\omega<\nu_{e, e f f} ., 7$ In the present work, a simplified two field $(\delta P, \delta \phi)$ nonlinear model of CTIM turbulence is derived by taking moments of the nonlinear bounceaveraged kinetic equations. The radial mode structure is obtained from a simple nonlocal model including equilibrium profile variations. Principal results of this paper include:

1. The thermal diffusivity at turbulent saturation is found to exhibit a nearly Bohm-like scaling. A new "neoclassical polarization density nonlinearity" 8 introduces an isotopedependent modification to this Bohm-like diffusivity.

2. The radial correlation length is predicted to be approximately the geometric mean of the trapped ion banana width and the equilibrium variation length $\left(L_{*}\right)$ of the diamagnetic drift frequency. Therefore, it is typically on the order of a couple of centimeters and decreases with increasing current.

3. The predicted density fluctuation level is lower than the conventional mixing length estimate, $\Delta r / L_{n}$. 
4. Ion temperature fluctuation level $\left(\delta T_{i} / T_{i}\right)$ is predicted to significantly exceed the density fluctuation level $\delta n / n_{0}$. The associated parallel velocity fluctuation level $\delta v_{i \|} / v_{T i}$, is shown to be negligibly small.

The remainder of this paper is organized as follows. In Sec. II, the new two-field nonlinear model for CTIM is derived from the nonlinear bounce-averaged drift kinetic equation. The linear properties of CTIM are discussed in Sec. III with emphasis on the distinctive broad radial mode structure. In Sec. IV, a steady state solution of the nonlinear equations is obtained from a one-point nonlinear analysis which treats the renormalized diffusivity as an eigenvalue. Finally, the fluctuation-driven thermal diffusivity as well as the various fluctuation levels at turbulent saturation are calculated. These results with a discussion of their implications for the tokamak confinement are presented in Sec. V.

\section{Theoretical Model}

In this section, a two-field fluid model which describes the nonlinear evolution of the collisionless trapped ion modes (CTIM) is derived. The starting point for this theoretical model is the bounce-averaged drift kinetic equations. For untrapped particles, both ion dynamics and electron dynamics are approximated by the Boltzmann response. The relevant frequencies in the linear analysis are ordered as $\omega_{t e}>\omega_{b e}>\omega, \omega_{* e}>\omega_{d e}>\nu_{e f f, e}$ and $\omega_{t i}>\omega_{b i}>\omega, \omega_{* i}>\omega_{d i}>\nu_{e f f, i}$. For untrapped particles, both ion dynamics and electron dynamics are approximated by the Boltzmann response. The trapped electron dynamics are described by the bounce-averaged nonlinear drift kinetic equation,

$$
\left(\frac{\partial}{\partial t}+i \omega_{D_{e}}\right) h_{e}-\frac{c}{B} \nabla\langle\delta \phi\rangle \times b \cdot \nabla h_{e}=i(2 \epsilon)^{1 / 2}\left(\omega-\omega_{* T e}(E)\right) \frac{e\langle\delta \phi\rangle}{T_{e}} F_{e},
$$

where $\delta f_{e}=\frac{e \delta \phi}{T_{e}} F_{M}+h_{e}$ defines the nonadiabatic part $\left(h_{e}\right)$ of the perturbed electron distribution function, $\delta f . F_{M}$ is the Maxwellian distribution function, $\omega_{* T e}(E)=\omega_{* e}\left\{1+\eta_{e}\left(E / T_{e}-\right.\right.$ $3 / 2)\}, \omega_{* e}=k_{\theta} \rho_{s} C_{s} / L_{n}, \eta_{e}=d \ln T_{e} / d l n n_{0}, \omega_{D e}$ is the energy dependent trapped electron precession drift frequency. For the trapped ions, the bounce-averaged nonlinear drift kinetic equation is similar to Eq.(1) with the most crucial difference being the appearance of the 
trapped-ion-orbit-averaged potential, $\langle\langle\delta \phi\rangle\rangle$.

$$
\left(\frac{\partial}{\partial t}+i \omega_{D_{i}}\right) g_{i}-\frac{c}{B} \nabla\langle\langle\delta \phi\rangle\rangle \times b \cdot \nabla g_{i}=-i(2 \epsilon)^{1 / 2} \tau\left(\omega-\omega_{* T i}(E)\right) \frac{e\langle\langle\delta \phi\rangle\rangle}{T_{e}} F_{i},
$$

It is well-known from the linear analysis that CTIM is a robust fluid-like instability with a linear growth rate which exceeds the real frequency. ${ }^{5}$ Accordingly, wave-particle resonant interactions will not be taken into account in the present analysis, and a moment approach will be adopted to facilitate the nonlinear analysis. Taking density and energy moments of Eqs. (1) and (2) leads to the following density and pressure evolution equations for trapped particle banana centers:

$$
\begin{gathered}
\left(\frac{\partial}{\partial t}-C_{s} \rho_{s} \nabla\langle\phi\rangle \times \boldsymbol{b} \cdot \nabla\right) \delta n_{e}=i(2 \epsilon)^{1 / 2}\left(\omega-\omega_{* e}\right)\langle\phi\rangle-i \omega_{d e} \delta P_{e} \\
\left(\frac{\partial}{\partial t}-C_{s} \rho_{s} \nabla\langle\phi\rangle \times \boldsymbol{b} \cdot \nabla\right) \delta P_{e}=\frac{3}{2} i(2 \epsilon)^{1 / 2}\left(\omega-\omega_{* p e}\right)\langle\phi\rangle,
\end{gathered}
$$

and

$$
\begin{gathered}
\left(\frac{\partial}{\partial t}-C_{s} \rho_{s} \nabla\langle\langle\phi\rangle\rangle \times b \cdot \nabla\right) \delta N_{i}=-i \tau(2 \epsilon)^{1 / 2}\left(\omega-\omega_{* i}\right)\langle\langle\phi\rangle\rangle-i \omega_{d i} \delta P_{i}, \\
\left(\frac{\partial}{\partial t}-C_{s} \rho_{s} \nabla\langle\langle\phi\rangle\rangle \times b \cdot \nabla\right) \delta P_{i}=-\frac{3}{2} i \tau(2 \epsilon)^{1 / 2}\left(\omega-\omega_{* p_{i}}\right)\langle\langle\phi\rangle\rangle .
\end{gathered}
$$

Here, $\phi \equiv e \delta \phi / T_{e}$. Note that $\delta n_{e} \equiv \int d^{3} v h_{\mathrm{e}} / n_{0}, \delta N_{i} \equiv \int d^{3} v g_{i} / n_{0}, \delta P_{e} \equiv \int d^{3} v h_{e} E / n_{0} T_{e}$, and $\delta P_{i} \equiv \int d^{3} v g_{i} E / n_{0} T_{i}$ come from the nonadiabatic parts of the trapped particle distributions only. These variables are therefore different from more conventional ones. ${ }^{9}$

In adopting the simplest nontrivial closure, the higher order velocity moments in Eqs.(4) and (6) have been neglected. As mentioned earlier, to the extent that wave-particle resonant interactions are not crucial, this simple approach captures the essence of the CTIM dynamics. Noting the similarities between the equations for trapped electrons and those for the trapped ions, the nonlinear system can be further simplified. A crucial difference between the trapped ion dynamics and the trapped electron dynamics is the finite (banana) orbit width effects of the trapped ions. Since the instabilities of interest here are long wavelength modes, the finite ion Larmor radius effects and the finite electron banana width effects are subdominant and can be ignored. Thus, in dealing with trapped electrons, the customary approximation for the bounce average can be utilized. This involves replacing the integration along the particle orbit by the integration along the appropriate magnetic field line. On the other 
hand, for trapped ions, the finite orbit excursion across the magnetic field line needs to be taken into account and is retained here by Taylor-expanding the perturbed quantity near the corresponding position along the reference magnetic field line. This leads to

$$
\langle\langle\phi\rangle\rangle \simeq\langle\phi\rangle+\frac{1}{2} \rho_{b i}^{2} \nabla_{\perp}^{2}\langle\phi\rangle
$$

where $\langle\phi\rangle$ is the potential value averaged along the field line. Subtracting Eq.(5) from Eq.(3), and using the quasi-neutrality condition,

$$
(1+\tau) \phi=\delta N_{i}-\delta n_{e}+\epsilon^{1 / 2} \rho_{b s}^{2} \nabla_{\perp}^{2}\langle\phi\rangle,
$$

leads to

$$
\begin{array}{r}
\frac{\partial}{\partial t}\left\{(1+\tau) \phi-2(2 \epsilon)^{1 / 2} \rho_{b s}^{2} \nabla_{\perp}^{2}\langle\phi\rangle\right\}+C_{s} \rho_{s}(2 \epsilon)^{1 / 2} \rho_{b s}^{2} \nabla\langle\phi\rangle \times b \cdot \nabla \nabla_{\perp}^{2}\langle\phi\rangle \\
+C_{s} \rho_{s} \rho_{b i}^{2} \nabla \delta N_{i} \times b \cdot \nabla \nabla_{\perp}^{2}\langle\phi\rangle=i\left|\omega_{d i}\right| \delta P-i \omega_{* e}(2 \epsilon)^{1 / 2} \rho_{b i}^{2} \nabla_{\perp}^{2}\langle\phi\rangle
\end{array}
$$

where $\delta P \equiv \delta P_{i}+\tau \delta P_{e}$ is the total nonadiabatic trapped particle pressure moment. In Eq.(7), the last term is a simplified form of the neoclassical polarization density which accounts for the difference between the actual trapped ion particle density and the density of banana centers. ${ }^{8}$ This can be shown to be closely related to the more familiar neoclassical polarization drift. ${ }^{10}$ Detailed analysis of the theoretical foundations for this neoclassical polarization density will be reported elsewhere. The last term in Eq.(8) is ignored for simplicity in the nonlinear analysis and the third term on the left hand side (LHS) is ignored as well, since a closely related term in Ion Temperature Gradient (ITG) Turbulence models has been shown to have no significant influence on the essential feature of results. ${ }^{11-15}$

Adding Eqs.(4) and (6) gives

$$
\left(\frac{\partial}{\partial t}-C_{s} \rho_{s} \nabla\langle\phi\rangle \times b \cdot \nabla\right) \delta P=-i \frac{3}{2} \tau(2 \epsilon)^{1 / 2}\left(\left|\omega_{* p i}\right|+\omega_{* p e}\right)\langle\phi\rangle-i \frac{3}{2} \tau(2 \epsilon)^{1 / 2}\left|\omega_{* p i}\right| \rho_{b i}^{2} \nabla_{\perp}^{2}\langle\phi\rangle,
$$

where the assumption, $|\omega|<\left|\omega_{* p i}\right|$, which will be justified later has been used. Equations (8) and (9) constitute a set of nonlinear equations which describe the evolution of CTIM's. It is interesting to compare the basic structure of this CTIM nonlinear model to the previous ITG ${ }^{11-15}$ or Resistive Pressure Gradient Turbulence Models. ${ }^{16}$ Apart from the coefficients 
related to the fraction of trapped particle population and to the banana width, the left hand side of Eq.(8) is similar to the Hasegawa-Mima equation ${ }^{17}$ or vorticity equation in the ITG turbulence model. The right hand side, on the other hand, describes the coupling to the pressure perturbation through curvature driven precession. Equation (9) is in a form familiar from Pressure Gradient Driven Turbulence Models which feature the $\mathbf{E} \times \mathbf{B}$ nonlinearity and the drive from the radial gradient of pressure. From these considerations, the CTIM can be expected to have an interchange-mode-like character.

\section{Linear Properties}

The key linear properties of CTIM are discussed in this section. In order to develop a systematic nonlinear theory of CTIM, the relevant results from the previous studies are incorporated in a simple context. In particular, results from studies using a comprehensive 2-D kinetic toroidal finite-element code show that under realistic conditions such as those in TFTR, the radial extent of the CTIM's is primarily governed by the spatial variations in the equilibrium profile gradients. ${ }^{4}$ This is in contrast to the previous conventional picture that long wavelength TIM's are radially localized between adjacent rational surfaces. ${ }^{18}$ These results also seem to be consistent with the BES measurements of long wavelength fluctuation properties in TFTR. ${ }^{3}$ Since the focus of this paper is on the nonlinear theory, a simple nonlocal model that captures the essential features of CTIM's mode structure is introduced. Incorporating these relevant features in a simple context allows development of a systematic but tractable nonlinear theory of CTIM.

Since studies of the 2-D global structure are beyond the scope of this paper, the following simplified radially nonlocal model is adopted. First, a nonlocal analysis is carried out near the region where the drive due to pressure gradient is maximum. ${ }^{19}$ This approach is also justified by the results in Ref. 4 . Hence, the equilibrium profile variation can be modeled by expanding the first term on the right hand side (RHS) of Eq.(9),

$$
\left(\left|\omega_{* p i}\right|+\omega_{* p e}\right) \simeq\left(\left|\omega_{* p i}\right|+\omega_{* p e}\right)(0)\left(1-X^{2} / L_{*}^{2}\right)
$$

where $X=0$ is chosen to be a radial location of the drive maximum, and $L_{*}$ is the scale length for the second radial derivative of the diamagnetic drift frequency. Away from this 
region, quantitative details of our approach cannot be applied directly. Although there exist some linear analyses supporting the broad radial stucture of the toroidal drift and ion temperature gradient driven modes, ${ }^{20-22}$ extension of the present nonlinear analysis to the region far away from the drive maximum is beyond the scope of this paper.

The perpendicular variation of perturbation is given by

$$
\nabla_{\perp}^{2}=\frac{\partial^{2}}{\partial X^{2}}-(n q / r)^{2}
$$

Then, linearization of Eqs. (8) and (9) leads to an eigenmode equation in the familiar form of the Weber's equation,

$$
\begin{aligned}
(2 \epsilon)^{1 / 2}\left(2 \omega^{2}+\omega\left|\omega_{* i}\right|\right. & \left.-\frac{3}{2} \tau\left|\omega_{d i} \omega_{* p i}\right|\right) \rho_{b s}^{2} \nabla_{\perp}^{2}\langle\phi\rangle=\omega^{2}(1+\tau)(1-\sqrt{2 \epsilon}) \phi \\
\cdot & +\frac{3}{2} \tau(2 \epsilon)^{1 / 2}\left|\omega_{d i}\right|\left(\left|\omega_{* p i}\right|+\omega_{* p e}\right)\left(1-X^{2} / L_{*}^{2}\right)\langle\phi\rangle .
\end{aligned}
$$

The reduction of the potential due to the bounce average along the field line will be ignored with $\langle\phi\rangle$ replaced by $\phi$ here. Effects of this approximation on both linear and nonlinear results are discussed in the Appendix. Note that the local growth rate is primarily determined from the balance between the first and the second terms on the RHS,

$$
\gamma_{0}^{2}=\frac{3 \tau(2 \epsilon)^{1 / 2}}{2(1+\tau)\left(1-(2 \epsilon)^{1 / 2}\right)}\left|\omega_{d i}\right|\left(\left|\omega_{* p i}\right|+\omega_{* p e}\right) .
$$

The eigenvalue of Eq.(10) contains a subdominant nonlocal correction as well as a finite banana width correction.

$$
\gamma^{2}=\gamma_{0}^{2}\left[1-\left\{1+\frac{1}{(1+\tau)(1-\sqrt{2 \epsilon})}\right\}\left(\sqrt{2 \epsilon} k_{\theta}^{2} \rho_{b_{s}}^{2}+\epsilon^{1 / 4} \rho_{b_{s}} / 2 L_{*}\right)\right] .
$$

This result is consistent with the assumption $|\omega|<\left|\omega_{* p i}\right|$. The lowest order radial eigenmode which has the highest linear growth rate is given by

$$
\phi_{n}(X)=\phi(0) e^{-X^{2} / \Delta r^{2}}
$$

where

$$
\begin{gathered}
\Delta r^{2}=C_{1} \rho_{b i} L_{*}, \\
C_{1}=\left\{\frac{2(2 \epsilon)^{1 / 2}}{(1+\tau)\left(1-(2 \epsilon)^{1 / 2}\right)}+\frac{1}{1+\tau\left(1+\eta_{e}\right) /\left(1+\eta_{i}\right)}\right\} .
\end{gathered}
$$




\section{Nonlinear Analysis}

In this section, a nonlinear analysis of Eqs. (8) and (9) is carried out to obtain the thermal diffusivity and various fluctuation levels at nonlinear saturation. In contrast to the familiar heuristic mixing length estimate, the renormalized transport coefficient is determined here as an eigenvalue necessary for the turbulent saturation. ${ }^{16,23}$ Since this procedure does not use an adjustible constant, it is more systematic and quantitative than more commonly used approaches. Equations (12) and (13) summarize the linear properties of CTIM's which are used in the following nonlinear calculations.

Using the standard weak coupling closure approximation, it is straightforward to renormalize the $\mathbf{E} \times \mathbf{B}$ nonlinearity of Eq. (9). Details of the renormalization procedure can be found in Ref. 16 and is not repeated here. This leads to the result,

$$
\left(-D \frac{\partial^{2}}{\partial X^{2}}+C k_{\theta}^{2}\right) \delta P=-\frac{3}{2} i \tau(2 \epsilon)^{1 / 2}\left(\left|\omega_{* p i}\right|+\omega_{* p e}\right)\left(1-X^{2} / L_{*}^{2}\right) \phi-\frac{3}{2} i \tau(2 \epsilon)^{1 / 2}\left|\omega_{* p i}\right| \rho_{b i}^{2} \nabla_{\perp}^{2} \phi,
$$

where $k_{\theta} \equiv n q / r$.

and

$$
D=\left(C_{s} \rho_{s}\right)^{2} \Sigma_{\boldsymbol{k}^{\prime}} \frac{k_{\theta}^{\prime 2}\left|\phi_{\boldsymbol{k}^{\prime}}\right|^{2}}{\Delta \omega_{\boldsymbol{k}^{\prime \prime}}}
$$

$$
C=\left(C_{s} \rho_{s}\right)^{2} \Sigma_{k^{\prime}} \frac{\left|\frac{\partial}{\partial X^{\prime}} \phi_{k^{\prime}}\right|^{2}}{\Delta \omega_{k^{\prime \prime}}}
$$

are the turbulent diffusivities for trapped particle pressure in the radial and perpendicular directions respectively. In arriving at this result, a standard procedure for renormalization of the $\mathbf{E} \times \mathbf{B}$ nonlinearity in the fluid context has been adopted. Specifically, the driven field $\phi_{\boldsymbol{k}^{\prime \prime}}^{(2)}$ which is the shielding contribution has been ignored, while keeping the bare nonlinearity, which is related to $\delta P_{k^{\prime \prime}}^{(2)}$. Although this approximation is adequate for Eq.(9), it is desirable to improve this renormalization scheme for Eq.(8).

It is convenient to adopt an approximation used in Ref. $16, \frac{\partial}{\partial X^{\prime}} \simeq \frac{k_{\theta}^{\prime} \partial}{k_{\theta}^{\prime \prime} \partial X^{\prime \prime}}$, to avoid the mathematical difficulty of explicitly calculating $\phi_{k^{\prime \prime}}^{(2)}$. Hence, the nonlinear term in Eq.(8) can be expressed as

$$
\begin{aligned}
\left(\nabla \phi \times b \cdot \nabla \nabla_{\perp}^{2} \phi\right)_{\boldsymbol{k}} \simeq & \frac{\partial}{\partial X} \Sigma_{\boldsymbol{k}^{\prime}}\left(-i k_{\theta}^{\prime}\right) \frac{k_{\theta}^{2}+2 k_{\theta} k_{\theta}^{\prime}}{k_{\theta}^{\prime \prime 2}} \phi_{-\boldsymbol{k}^{\prime}}\left(\nabla_{\perp}^{2} \phi_{\boldsymbol{k}^{\prime \prime}}\right)^{(2)} \\
& -i k_{\theta} \Sigma_{\boldsymbol{k}^{\prime}}\left(\frac{k_{\theta}^{2}+2 k_{\theta} k_{\theta}^{\prime}}{k_{\theta}^{\prime \prime 2}}\right) \frac{\partial \phi_{-} \boldsymbol{k}^{\prime}}{\partial X^{\prime}}\left(\nabla_{\perp}^{2} \phi_{\boldsymbol{k}^{\prime \prime}}\right)^{(2)}
\end{aligned}
$$


Equation.(8) is then renormalized by substituting the expression for the driven vorticity $\left(\nabla_{\perp}^{2} \phi_{\mathbf{k}^{\prime \prime}}\right)^{(2)}$, to obtain

$$
\rho_{b s}^{2}\left\{-k_{\theta}^{2} E \phi_{k}+\frac{\partial}{\partial X} F \frac{\partial}{\partial X} \phi_{k}-k_{\theta}^{2} G \nabla_{\perp}^{2} \phi_{k}+\frac{\partial}{\partial X} H \frac{\partial}{\partial X} \nabla_{\perp}^{2} \phi_{k}\right\}=i\left|\omega_{d i}\right| \delta P_{k},
$$

where the various renormalized transport coefficients are given by

$$
\begin{gathered}
H=\left(C_{s} \rho_{s}\right)^{2} \Sigma_{\boldsymbol{k}^{\prime}}\left(\frac{k_{\theta}^{\prime}}{k_{\theta}^{\prime \prime}}\right)^{2} k_{\theta}^{\prime 2}\left|\phi_{\boldsymbol{k}^{\prime}}\right|^{2} / \Delta \omega_{\boldsymbol{k}^{\prime \prime}}, \\
G=\left(C_{s} \rho_{s}\right)^{2} \Sigma_{\boldsymbol{k}^{\prime}}\left(\frac{k_{\theta}^{\prime}}{k_{\theta}^{\prime \prime}}\right)^{2}\left|\frac{\partial \phi_{\boldsymbol{k}^{\prime}}}{\partial X^{\prime}}\right|^{2} / \Delta \omega_{\boldsymbol{k}^{\prime \prime}}, \\
F=\left(C_{s} \rho_{s}\right)^{2} \Sigma_{\boldsymbol{k}^{\prime}}\left(\frac{k_{\theta}^{\prime}}{k_{\theta}^{\prime \prime}}\right)^{2} k_{\theta}^{\prime 2}\left|\nabla_{\perp} \phi_{\boldsymbol{k}^{\prime}}\right|^{2} / \Delta \omega_{\boldsymbol{k}^{\prime \prime}},
\end{gathered}
$$

and

$$
E=\left(C_{s} \rho_{s}\right)^{2} \Sigma_{\boldsymbol{k}^{\prime}}\left(\frac{k_{\theta}^{\prime}}{k_{\theta}^{\prime \prime}}\right)^{2}\left|\frac{\partial \nabla_{\perp} \phi_{\boldsymbol{k}^{\prime}}}{\partial X^{\prime}}\right|^{2} / \Delta \omega_{\boldsymbol{k}^{\prime \prime}}
$$

$\mathrm{H}$ and $\mathrm{G}$ are the turbulent diffusivities for the vorticity in the radial and perpendicular directions respectively, while $\mathrm{F}$ and $\mathrm{E}$ describe the self-consistent turbulent back-reaction of the convecting velocity on $\phi_{\boldsymbol{k}}$ evolution. Considering a long wavelength test mode such that $k_{\perp}^{2} \ll k_{\perp}^{2}$, we note that the first two terms on the LHS of Eq.(16) are larger than the last two terms. If only the two dominant nonlinear terms in Eq.(16) are retained, Eq.(14) yields, $\frac{3}{2}(2 \epsilon)^{1 / 2}\left|\omega_{d i}\right|\left\{\tau\left(\left|\omega_{* p i}\right|+\omega_{* p e}\right)\left(1-X^{2} / L_{*}^{2}\right)+\left|\omega_{* p i}\right| \rho_{b s}^{2} \nabla_{\perp}^{2}\right\} \phi=\rho_{b s}^{2}\left(C k_{\theta}^{2}-D \frac{\partial^{2}}{\partial X^{2}}\right)\left(F \frac{\partial^{2}}{\partial X^{2}}-E k_{\theta}^{2}\right) \phi$.

By Fourier transforming, this fourth order differential equation in configuration space can be reduced to the following second order differential equation,

$$
\begin{array}{r}
-\frac{3}{2}(2 \epsilon)^{1 / 2} \tau\left|\omega_{d i}\right|\left(\left|\omega_{* p i}\right|+\omega_{* p e}\right) L_{*}^{-2} \frac{\partial^{2}}{\partial u^{2}} \phi(u)=\frac{3}{2}(2 \epsilon)^{1 / 2}\left|\omega_{d i}\right|\left\{\tau\left(\left|\omega_{* p i}\right|+\omega_{* p e}\right)\right. \\
\left.-\rho_{b s}^{2}\left|\omega_{* p i}\right|\left(u^{2}+k_{\theta}^{2}\right)\right\} \phi(u)-k_{\theta}^{2} \rho_{b s}^{2}\left\{(C F+D E) u^{2}+C E k_{\theta}^{2}\right\} \phi(u) .
\end{array}
$$

Here, $O\left(u^{4}\right)$ term on the RHS has been ignored since the physically relevant turning point is determined by the constant and $O\left(u^{2}\right)$ terms in the effective potential. On the RHS of Eq.(18), the first term describes the pressure gradient drive coupled to bad curvature. Reduction of the potential due to the finite banana width effects is included here. The last term is the effective nonlinear damping of the test mode $k$ due to the nonlinear mode 
coupling. Since it is expected that $D \gg \gamma_{0} \rho_{b s}^{2}$, the $u$-dependent nonlinear term dominates over the finite banana width reduction of the potential (i.e., $u$-dependent linear term on the RHS).

The thermal diffusivity $\mathrm{D}$ as an eigenvalue of Eq.(18) can now be obtained, by expressing other transport coefficients in terms of $D$; i.e.,

$$
\begin{gathered}
C \simeq \frac{\left.<k_{r}^{\prime 2}\right\rangle}{\left.<k_{\theta}^{\prime 2}\right\rangle} D \simeq \frac{k_{r}^{2}}{k_{\theta}^{2}} D, \\
F \simeq \frac{\left.<k_{\theta}^{\prime 2}\right\rangle}{\left\langle k_{\theta}^{\prime \prime 2}\right\rangle} k_{\theta}^{2} D \simeq k_{\theta}^{2} D,
\end{gathered}
$$

and

$$
E \simeq \frac{\left.<k_{r}^{\prime 2}\right\rangle}{\left\langle k_{\theta}^{\prime \prime 2}\right\rangle} k_{\theta}^{2} D \simeq k_{r}^{2} D
$$

For the lowest radial eigenfunction,

$$
k_{\theta}^{2} \rho_{b s}^{2} k_{r}^{4} D^{2}-\gamma_{*}^{2}=-\frac{\gamma_{0}}{\sqrt{2} L_{*}} k_{\theta} \rho_{b s} k_{r} D
$$

where

$$
\gamma_{*}^{2} \equiv \gamma_{0}^{2}\left[1-\frac{k_{\theta}^{2} \rho_{b i}^{2}}{\left.\left\{1+\tau\left(1+\eta_{e}\right) /\left(1+\eta_{i}\right)\right)\right\}}\right] .
$$

Since the RHS is an $O\left(\rho_{b i} / L_{*}\right)$ correction to the dominant terms on the LHS, Eq.(19) yields,

$$
D \simeq \frac{\gamma_{*}}{k_{r}^{2} k_{\theta} \rho_{b s}}\left(1-\frac{1}{\sqrt{2}} \frac{\gamma_{0}}{\gamma_{*}} \frac{1}{k_{r} L_{*}}\right)^{1 / 2}
$$

Using Equations (11),(13), and (20) can now be combined to give the one-fluid thermal diffusivity,

$$
\chi \simeq D \simeq \frac{c T_{i}}{e B_{\phi}} \frac{L_{*}}{\sqrt{R}}\left(\frac{1}{L_{p i}}+\frac{\tau}{L_{p e}}\right)^{1 / 2}\left(1-\frac{1}{\Gamma_{*}} \sqrt{\frac{C_{1} \rho_{b i}}{2 L_{*}}}\right)^{1 / 2} C_{2} \Gamma_{*}
$$

where

$$
\Gamma_{*} \equiv\left[1-\frac{k_{\theta}^{2} \rho_{b i}^{2}}{1+\tau\left(1+\eta_{e}\right) /\left(1+\eta_{i}\right)}\right]^{1 / 2}, C_{2}=\sqrt{\frac{2(2 \epsilon)^{1 / 2}}{2(1+\tau)(1-\sqrt{2 \epsilon})}} C_{1},
$$

$C_{1}$ is defined at the end of Sec. III.

It is of interest here to examine to what extent the result in Eq.(21) is a consequence 
of the specific renormalization procedure used here and the new neoclassical polarization nonlinearity. From dimensional considerations such as random walk arguments, the thermal diffusivity can be expected to scale as

$$
\chi \simeq(\Delta r)^{2} \gamma_{0} \simeq \frac{c T_{i}}{e B_{\phi}} \frac{L_{*}}{\sqrt{R}}\left(\frac{1}{L_{p i}}+\frac{\tau}{L_{p e}}\right)^{1 / 2} k_{\theta} \rho_{b i},
$$

where use has been made of Eqs.(11) and (13). Therefore, Bohm-like scaling can be recovered from Eq.(21) by assuming $k_{\theta} \rho_{b i} \simeq$ const. Of course, in the context of the one-point renormalization procedure considered in this paper, an analytical prediction for the saturated spectrum cannot be obtained. With regard to the role of the neoclassical polarization nonlinearity, which contains one more factor of $\left(k_{\theta} \rho_{b i}\right)^{2}$ compared to the $E \times B$ nonlinearity, the main points to emphasize are that:

i) It introduces an isotope dependent term $\sqrt{\frac{p_{b i}}{L_{*}}}$ in Eq.(21); and

ii) Bohm scaling in Eq.(21) can be obtained without assuming $k_{\theta} \rho_{b i} \simeq$ constant. This is obvious from Eq. (20), since $\gamma_{*} \propto k_{\theta}$.

In estimating the fluctuation levels from the present analysis, the definition of $D$ given below Eq.(14) can be combined with the additional assumption, $\Delta \omega_{\mathbf{k}^{\prime \prime}} \simeq \gamma_{0}$, to give:

$$
D \simeq \frac{c T_{i}}{e B_{\phi}} k_{\theta} R^{1 / 2}\left(\frac{1}{L_{p i}}+\frac{\tau}{L_{p e}}\right)^{-1 / 2}|\phi|^{2} .
$$

If this relation is equated to Eq.(21) and the approximation, $k_{\theta} \rho_{b i} \simeq$ const is used, then

$$
\frac{e \delta \phi}{T_{e}} \simeq \frac{\Delta r}{\sqrt{R L_{p}}}
$$

The trapped ion pressure fluctuation level at saturation is then obtained from Eq.(6) by balancing the $E \times B$ convective nonlinearity (the second term on the LHS) with the pressure gradient drive (the second term on the RHS); i.e.,

$$
\left|\frac{\delta P_{i}}{P_{i}}\right| \simeq \sqrt{\frac{R}{L_{p}}} \frac{e \delta \phi}{T_{e}} \simeq \frac{\Delta r}{L_{p}}
$$

The trapped electron density fluctuation level at saturation is similarly obtained from Eq.(3) by the balance between the second term on the LHS and the second term on the RHS. Combining with the adiabatic response then yields,

$$
\frac{\delta n}{n_{0}} \simeq\left\{1-\frac{i}{1+\eta_{i}} \sqrt{\frac{r}{R}}\left(\frac{R}{L_{*}}\right)^{3 / 4}\right\} \frac{e \delta \phi}{T_{e}} .
$$


Finally, the present analysis indicates that the parallel ion velocity perturbation is negligible. This is a consequence of the fact that the untrapped particle dynamics are dominantly adiabatic due to the slow evolution of the mode.

\section{Discussions}

In this paper, a nonlinear theory of collisionless trapped ion modes is presented. As indicated by Eq.(21), the thermal diffusivity exhibits a nearly Bohm-like scaling similar to trends observed in the $\rho_{*}$-scan experiment results on TFTR. ${ }^{1}$ The aspect ratio dependence contained in $C_{1}$ and $C_{2}$ are typical of trapped-particle-driven-turbulence. ${ }^{6,7,24,25}$ With regard to isotope scaling, the ion mass dependence contained in the $\sqrt{\frac{\rho_{b i}}{L_{*}}}$ factor originates from the new nonlinearity in Eq.(8) associated with the neoclassical polarization density. Although confinement is accordingly expected to improve with larger ion mass, the predicted trend here is not nearly as strong as that reported in the TFTR DT-experiments. ${ }^{26}$ It is important to keep in mind that these results are applicable only for high temperature, low collisionality plasmas such as encountered in TFTR. This point may also be relevant to the interpretation of recent nonlinear global gyrokinetic simulation results ${ }^{27}$ where deviation from Bohm scaling becomes larger with decreasing system size.

The radial correlation length given below Eq. (13) is determined from the balance between the finite banana orbit width reduction of the fluctuation potential and the radial variation of equilibrium profiles. Since it decreases with increasing plasma current $\left(I_{p}\right)$, it is in qualitative agreement with recent BES measurements on TFTR. ${ }^{28}$ Regarding fluctuation amplitude characteristics, the density fluctuation at saturation is found to be smaller than the simple mixing length estimate $\Delta r / L_{n}$ and therefore again in agreement with trends observed in the BES studies. ${ }^{28}$ It is particularly important to note that the predicted ion temperature fluctuation level can easily exceed $\delta n / n_{0}$. This is in agreement with preliminary results from the high-frequency charge exchange recombination spectroscopy (HF-CHERS) measurements on TFTR. ${ }^{29}$ 


\section{Acknowledgments}

The authors would like to thank M.A. Beer, Liu Chen, P.H. Diamond, J.F. Drake, W.W. Lee, S.E. Parker, S.F. Paul, G. Rewoldt, and S.D. Scott for useful discussions. This work was supported by the U.S. Department of Energy Contract No. DE-AC02-76-CHO-3073.

\section{Appendix: Effects of Bounce Average along the Field Line}

In this Appendix, the effect of the reduction of the potential due to bounce average along the field line is briefly discussed. As shown in Ref. 24, the trapped particles respond to the bounce average of the projection of the potential fluctuation along the magnetic field line. Therefore, due to the difference between the pitch of magnetic field line and that of the mode, the trapped particle response is localized near the mode rational surface roughly within $\Delta_{n} \equiv(n d q / d r)^{-1}$. Hence, the analysis presented in the main text which ignores this effect is justified when $\Delta r<\Delta_{n}$. If typical parameters in the core region of TFTR are considered, this corresponds to $n<\frac{r}{q s \Delta r} \simeq \frac{40 \mathrm{~cm}}{2 \times 0.5 \times 2 \mathrm{~cm}} \simeq 20$, where $s$ is the magnetic shear. It is also interesting to observe that the requirement for a fluctuation to be in the trapped ion regime $\omega<\omega_{b i}$, can be written as an upper bound on the toroidal mode number $n$,

$$
n<\frac{r \epsilon^{1 / 2}}{q^{2}}\left(\frac{1}{L_{p i}}+\frac{\tau}{L_{p e}}\right)^{-1 / 2} R^{-1 / 2} L_{*}(\Delta r)^{-2} .
$$

This condition is easier to be satisfied than $\Delta r<\Delta_{n}$.

Next, a rough estimate is given for the effect of the bounce average reduction of the potential in the opposite limit, $\Delta r>\Delta_{n}$, which is applicable to a relatively shorter wavelength regime. It is convenient to follow a procedure similar to that in Ref. 24. The main difference is that, while the trapped electron modes considered in Ref. 24 are either dissipative or resonant instabilities, the CTIM is a reactive fluid-like instability. Constructing a quadratic form of Eq. (10), it is seen that the bounce-average-modified local growth rate $\gamma_{B A}$ is again primarily determined from the balance between the first and the second terms on the RHS,

$$
\gamma_{B A}^{2} \sim \gamma_{0}^{2} \frac{\Delta_{n}}{\Delta r} \ln \left(\frac{\Delta r}{\Delta_{n}}\right) \sim \gamma_{0}^{2} \frac{1}{s} \sqrt{\frac{\rho_{b i}}{L_{*}}} .
$$


Here, a weaker logarithmic dependence is ignored, and $k_{\theta} \rho_{b i} \sim$ const is used in the absence of an analytical prediction of the saturated spectrum. Consequently, the thermal diffusivity scaling changes to

$$
\chi_{B A} \sim \chi \frac{1}{s^{1 / 2}}\left(\frac{\rho_{b i}}{L_{*}}\right)^{1 / 4}
$$

The thermal diffusity now exhibits a gyro-reduced Bohm scaling to the quarter power. This scaling is still closer to Bohm rather than gyro-Bohm even though an asymptotic expression which is valid for high mode number $(n \gg r / q s \Delta r)$ perturbation has been used. Therefore, it can be concluded that the simple model presented in the main text illustrates the essential feature of CTIM turbulence with the minimum mathematical complexity. 


\section{References}

${ }^{1}$ F.W. Perkins, C.W. Barnes, D.W. Johnson, S.D. Scott, M.C. Zarnstorf, M.G. Bell, R.E. Bell, C.E. Bush, B. Grek, K.W. Hill, D.K. Mansfield, H. Park, A.T. Ramsey, J. Schivell, B.C. Stratton, and E. Synakowski, Phys. Fluids B 5, 477 (1993).

${ }^{2}$ C.C. Petty, T.C. Luce, R.I. Pinsker, K.H. Burrell, S.C. Chiu, P. Gohil, R.A. James, and D. Wroblewski, Phys. Rev. Lett. 74, 1763 (1995).

${ }^{3}$ R.J. Fonck, G. Cosby, R.D. Durst, S.F. Paul, N. Bretz, S. Scott, E. Synakowski, and G. Taylor, Phys. Rev. Lett. 70, 3736 (1993).

${ }^{4}$ W.M. Tang and G. Rewoldt, Phys. Fluids B 5, 2451 (1993).

${ }^{5}$ B.B. Kadomtsev and O.P. Pogutse, Sov. Phys. JETP 24, 1172 (1967).

${ }^{6}$ P.H. Diamond and H. Biglari, Phys. Rev. Lett. 65, 2865 (1990).

${ }^{7}$ H. Biglari and P.H. Diamond, Phys. Fluids B 3, 1797 (1991).

${ }^{8}$ B.H. Fong and T.S. Hahm, Private Communications (1995).

${ }^{9}$ M.A. Beer, Ph. D. Thesis, Princeton University, 1995.

${ }^{10}$ F.L. Hinton and J.A. Robertson, Phys. Fluids 27, 1243 (1984):

${ }^{11}$ W. Horton, R.D. Estes, and D. Biskamp, Plasma Phys. 22, 663 (1980).

${ }^{12}$ W. Horton, D.-I. Choi, and W.M. Tang, Phys. Fluids 24, 1077 (1981).

${ }^{13}$ G.S. Lee and P.H. Diamond, Phys. Fluids 29, 3291 (1986).

${ }^{14}$ J.W. Connor, Nucl. Fusion 26, 193 (1986).

${ }^{15}$ W.W. Lee and W.M. Tang, Phys. Fluids 31, 612 (1988).

${ }^{16}$ B.A. Carreras, L. Garcia, and P.H. Diamond, Phys. Fluids 30, 1388 (1987).

${ }^{17}$ A. Hasegawa and K. Mima, Phys. Rev. Lett. 39, 205 (1977). 
${ }^{18}$ N.T. Gladd and D.W. Ross, Phys. Fluids 16, 1706 (1973).

${ }^{19}$ N.A. Krall and M.N. Rosenbluth, Phys. Fluids 8, 1488 (1965).

${ }^{20}$ J.W. Connor, J.B. Taylor, and H.R. Wilson, Phys. Rev. Lett. 70, 1803 (1993).

${ }^{21}$ F. Romanelli and F. Zonca, Phys. Fluids B 5, 4081 (1993).

${ }^{22}$ J.Y. Kim and M. Wakatani, Phys. Rev. Lett. 73, 2200 (1994).

${ }^{23}$ S.I. Itoh, K. Itoh, A. Fukuyama, and M. Yagi, Phys. Rev. Lett. 72, 1200 (1994).

${ }^{24}$ F.Y. Gang, P.H. Diamond, and M.N. Rosenbluth, Phys. Fluids B 3, 68 (1991).

${ }^{25}$ T.S. Hahm and W.M. Tang, Phys. Fluids B 3, 989 (1991).

${ }^{26}$ S.D. Scott, M.C. Zarnstorff, Cris W. Barnes, R. Bell, N.L. Bretz, C. Bush, Z. Chang, D. Ernst, R.J. Fonck, L. Johnson, E. Mazzucato, R. Nazikian, S. Paul, J. Schivell, E.J. Synakowski, H. Adler, M. Bell, R. Budny, E. Fredrickson, B. Grek, A. Janos, D. Johnson, D. McCune, H. Park, A. Ramsey, M.H. Redi, G. Taylor, M. Thompson, and R. Wieland, Phys. Plasmas 2, 2299 (1995).

${ }^{27}$ S.E. Parker, J.C. Cummings, W.W. Lee, and H.E. Mynick, in Theory of Fusion Plasmas 1994, edited by E. Sindoni, F. Troyon, and J. Vaclavik (Societa Italiana di Fisica/Editrice Compositori, Bologna, Italy, August 1994), p. 219.

${ }^{28}$ S.F. Paul, R.D. Durst, R.J. Fonck, S.M. Grossman, J.S. Kim, S.D. Scott, and the TFTR Group, Private Communications (1995).

${ }^{29}$ H.T. Evenson, R.D. Durst, R.J. Fonck, S.F. Paul, and M.C. Zarnstorff, Private communications (1995).

\section{DISCLAIMER}

This report was prepared as an account of work sponsored by an agency of the United States Government. Neither the United States Government nor any agency thereof, nor any of their employees, makes any warranty, express or implied, or assumes any legal liability or responsibility for the accuracy, completeness, or usefulness of any information, apparatus, product, or process disclosed, or represents that its use would not infringe privately owned rights. Reference herein to any specific commercial product, process, or service by trade name, trademark, manufacturer, or otherwise does not necessarily constitute or imply its endorsement, recommendation, or favoring by the United States Government or any agency thereof. The views and opinions of authors expressed herein do not necessarily state or reflect those of the United States Government or any agency thereof. 